\title{
Correction: Morning blood pressure surge in young black and white adults: The African-PREDICT Study
}

\author{
Gontse Gratitude Mokwatsi $\mathbb{C}^{1} \cdot$ Aletta Elisabeth Schutte $\mathbb{I}^{1,2} \cdot$ Catharina Martha Cornelia Mels $\mathbb{D}^{1,2}$ • \\ Ruan Kruger (D) ${ }^{1,2}$
}

Published online: 19 November 2018

(c) Springer Nature Limited 2018

Correction to: Journal of Human Hypertension 32 (2018); https://doi.org/10.1038/s41371-018-0089-3; published online 23 July 2018

In this article, the authors regret that they mentioned erroneously that none of their study participants had an exaggerated morning blood pressure surge. They would like to point out that 40 participants in their study population had an exaggerated sleep-trough surge, whereas 128 had an exaggerated dynamic surge.

The authors apologise for any inconvenience this may have caused.
Ruan Kruger

ruan.kruger@me.com

1 Hypertension in Africa Research Team (HART), North-West University, Potchefstroom, South Africa

2 Medical Research Council: Research Unit for Hypertension and Cardiovascular Disease, Faculty of Health Sciences, North-West University, Potchefstroom, South Africa 